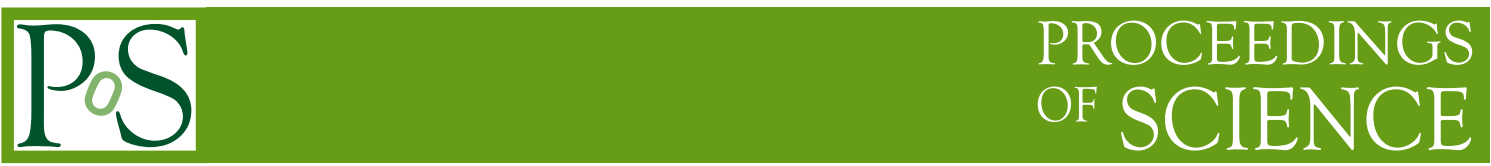

\title{
Detecting AGNs Using Multi-filter Imaging Data
}

\author{
X.Y. Dong and M.M. De Robertis * \\ York University, Canada \\ E-mail: xydong@yorku.ca, mmdr@yorku.ca
}

\begin{abstract}
We present a technique to detect AGNs using ugriz imaging data and via carefully conducted aperture photometric analysis. To optimize the process, each filter must have the same Point Spread Function (PSF) whose width is as sharp as possible. We applied an imaging manipulating algorithm, shapelets, to each galaxy image which allowed the reconstructed images to have the same PSF while enhancing the spatial resolution. We also designed artificial neural networks (ANNs) to estimate photometric redshifts, assign a morphological $T$ type to each galaxy, and determine whether a galaxy is an AGN, starburst(SB), star-forming (SF), or passive (absorption-line) system. The ANN correctly identified $67 \%$ of AGNs and $94 \%$ of all SBs/SFs. The results indicated that in the local Universe $(z \leq 0.1)$ AGNs and passive galaxies prefer early-type (elliptical and early spiral) galaxies, while $\mathrm{SBs} / \mathrm{SFs}$ were more often found in late-type spirals.
\end{abstract}

Nuclei of Seyfert galaxies and QSOs - Central engine \& conditions of star formation November 6-8, 2012

Max-Planck-Insitut für Radioastronomie (MPIfR), Bonn, Germany

${ }^{*}$ The authors gratefully wish to acknowledge support from the Natural Sciences and Engineering Research Council of Canada for this program. 


\section{Motivation}

AGN, starburst and passive galaxies are somewhat distinguishable on a color-color diagram because their Spectral Energy Distributions (SEDs) are inherently different, although lower-luminosity AGNs are more difficult to separate from passive galaxies because of host contamination. Photometric analyses on simulated galaxy images indicate that identification of galaxy type is significantly enhanced if each filter has the same PSF. Moreover, AGNs can be identified more easily when images possess sharper PSFs (within reason). The aim of this project is to develop a technique to detect AGNs using multi-filter imaging data and aperture photometric analysis.

\section{Two-step shapelets routine}

The shapelets algorithm [2,3] decomposes a galaxy image using Gaussian-weighted Hermite polynomials as basis functions. The shapelets coefficients can be used to reconstruct an observed image (with the PSF) or an intrinsic image (without the PSF). By reconstructing each galaxy using an optimized PSF (FWHM=3 pixels), shapelets improves an image's spatial resolution and adjusts images taken with different filters to have the same PSF. In order to improve shapelets decomposition of high Sérsic index galaxies, we adopted a two-step approach: apply shapelets using basis functions of low order numbers, followed by a second application on the remaining image. Both are combined to generate the final reconstructed image.

\section{Data selection and reduction}

This technique was applied to a data set selected from the Canada France Hawaii Telescope Legacy Survey (CFHTLS); in particular, the wide synoptic survey W3 and W4 from data releases 5 and 6 . Highly elongated objects or galaxies with high internal extinction were avoided by selecting only galaxies with inclinations $\leq 60^{\circ}$ in $r$. The galaxy magnitude in $u$ was required to be brighter than +22 mag for a reliable shapelets decomposition. The final sample included about 9000 galaxies.

shapelets decomposition was applied on each galaxy's ugriz images. A galaxy image was reconstructed using a 2-D Gaussian PSF with FWHM=3 pixels. The photometric measurements were carried out on the reconstructed image. The photometric parameters obtained included flux, flux radii, concentration index, color, color gradients and surface brightness.

\section{Analysis of the photometric parameters using the artificial neural networks}

ANN is often used in astronomy for galaxy morphological classification and photometric redshift estimation with large data sets. We also used it for pattern recognition in order to identify galaxies as either an AGN, a starburst(SB)/star-forming (SF), or a passive galaxy. The parameters used for ANN trainings included galaxy magnitudes in $u, g, r, i, z$; galaxy colors $u-g, g-r, r-i$, $i-z$; flux radii in $r$; concentration index in $r$; mean surface brightness within $50 \%$ Petrosian flux radius in $r$; and color gradients $\Delta(u-r)$ and $\Delta(g-r)$. 
Estimate photometric redshift. The ANN training sample was selected from the SDSS DR7 Main Galaxy Catalog that overlaps with CFHTLS W3 and W4. When $z \leq 0.4$, the rms between the spectroscopic redshift given by SDSS and the photometric redshift by ANN is 0.026.

Assign morphological $T$ type. The training sample was selected from a visual classification by Nair and Abraham[4] that overlaps with W3 and W4. The resulting rms is $1.6 T$ types.

Identify AGN, SB/SF, and passive galaxies. The training sample was selected from the MPA/JHU DR7 [5]. ANN identified 67\% of AGNs, 63\% passive galaxies, and 94\% SBs/SFs with confidence.

\section{Results}

We confine our discussions to galaxies with $z \leq 0.1$, because some parameters used for ANN require sufficient spatial resolution.

Galaxy morphological $T$ type. Based on ANN results, within $z=0.1, u \leq+22 \mathrm{mag}$, the galaxy morphology is mostly evenly distributed from lenticulars $(T=-2)$ to late type spirals $(T=5)$.

AGN, SB/SF, and passive galaxies. Within $z=0.1$, we identified 186 out of 1677 galaxies as AGNs using ANN; comparable to the number of MPA/JHU identified AGNs (60 out of 533). The mean absolute magnitudes of AGN and passive galaxies are similar with AGNs being slightly brighter. SBs/SFs are 1-2 magnitudes fainter. Both AGN and passive galaxies prefer early type galaxies, while SBs/SFs prefer late spirals as showed in Figure 1.

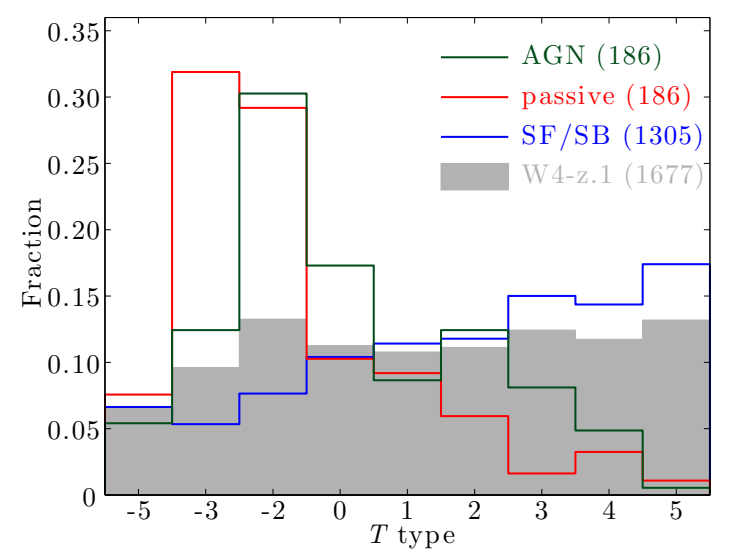

Figure 1: Normalized $T$ type distributions of each spectral class. The number within the parentheses is the number of galaxies within each class.

\section{References}

[1] V.L. Sarajedini, R.F. Green, and R.E. Griffiths et al. 1999, ApJS, 121, 417

[2] A. Réfrégier, 2003, MNRAS, 338, 35

[3] A. Réfrégier and D. Bacon, 2003, MNRAS, 338, 48

[4] P.B. Nair and R.G. Abraham, 2010, ApJS, 186, 427

[5] C.A. Tremonti, T.M. Heckman, and G. Kauffmann et al., 2004, AJ, 613, 898 